\title{
Paper-Based Surfaces with Extreme Wettabilities for Novel, Open-Channel Microfluidic Devices
}

\author{
Chao Li, Mathew Boban, Sarah A. Snyder, Sai P. R. Kobaku, Gibum Kwon, \\ Geeta Mehta,* and Anish Tuteja*
}

In this work, a facile methodology is discussed, involving fluoro-silanization followed by oxygen plasma etching, for the fabrication of surfaces with extreme wettabilities, i.e., surfaces that display all four possible combinations of wettabilities with water and different oils: hydrophobic-oleophilic, hydrophilic-oleophobic, omniphobic, and omniphilic. Open-channel, paper-based microfluidic devices fabricated using these surfaces with extreme wettabilities allow for the localization, manipulation, and transport of virtually all high- and low-surface tension liquids. This in turn expands the utility of paper-based microfluidic devices to a range of applications never before considered. These include, as demonstrated here, continuous oil-water separation, liquid-liquid extraction, open-channel microfluidic emulsification, microparticle fabrication, and precise measurement of mixtures' composition. Finally, the biocompatibility of the developed microfluidic devices and their utility for cell patterning are demonstrated.

\section{Introduction}

Surfaces exhibiting extreme wettabilities (Figure 1a), hydrophobic (HP)/oleophobic (OP) (omniphobic, all liquids nonwetting), ${ }^{[1]}$ $\mathrm{HP} /$ oleophilic (OL) (water nonwetting, oil wetting), ${ }^{[2]}$ hydrophilic

Dr. C. Li, S. A. Snyder, Dr. G. Kwon, Prof. G. Mehta, Prof. A. Tuteja

Department of Materials Science and Engineering

University of Michigan

Ann Arbor, MI 48109, USA

E-mail: mehtagee@umich.edu; atuteja@umich.edu

M. Boban, Dr. S. P. R. Kobaku, Prof. G. Mehta,

Prof. A. Tuteja

Department of Macromolecular Science and Engineering

University of Michigan

Ann Arbor, MI 48109, USA

Prof. G. Mehta

Department of Biomedical Engineering

University of Michigan

Ann Arbor, MI 48109, USA

Prof. A. Tuteja

Department of Chemical Engineering

University of Michigan

Ann Arbor, MI 48109, USA

Prof. A. Tuteja

Biointerfaces Institute

University of Michigan

Ann Arbor, MI 48109, USA

DOI: 10.1002/adfm.201601821
(HL)/OP (water wetting, oil nonwetting), ${ }^{[3]}$ and HL/OL (omniphilic, all liquids wetting),${ }^{[4]}$ have a large number of applications including chemical and biological protection, oil-water separation, stain-resistant textiles, "non-stick" coatings, controlling protein and cell adhesion on surfaces, reduction of biofouling, and enhanced heat transport. However, there is no established technique that allows for selectively generating and patterning all four extreme wettabilities $^{[5]}$ on a single surface, especially at the length scale necessary for microfluidic control. In this work, we discuss a facile methodology for the fabrication of surfaces with extreme wettabilities by selectively modifying the surface energy and roughness of different paper surfaces.

Paper has recently emerged as a promising materials platform for microfluidic devices due to its low cost, easy disposal, high surface area, capillary-based wetting, flexibility, and compatibility with a wide range of patterning and printing techniques. ${ }^{[6]}$ Since the first report of using paper as a base material in microfluidics by Whitesides et al. in 2007, ${ }^{[7]}$ a new era of paper-based microfluidic devices has arisen. ${ }^{[8]}$ The ability to pattern wetting/non-wetting channels on paper has allowed multiplexed, small-volume fluid control both in 2D lateral flow on a single surface ${ }^{[9]}$ and 3D flow on stacked layers connected through pores. ${ }^{[10]}$

Generally, fluidic channels introduced on paper surfaces are composed of wettable domains bounded by non-wettable domains, or by air gaps. ${ }^{[11]}$ In most cases, paper-based microfluidic channels have been developed to contain only water or aqueous solutions. ${ }^{[12]}$ Few reported techniques used for generating patterned wettability on paper result in devices compatible with even a limited number of non-aqueous liquids. ${ }^{[13]}$ Further, the wettable channels in the paper-based microfluidic systems reported thus far show no selective wettability with liquids possessing different surface tensions and/or polarities. In other words, all liquids wet these fluidic channels. Overall, there is no established technique that allows for the selective generation of all four "extreme wettabilities" ${ }^{[5]}$ on paper-based microfluidic channels; that is, the four possible combinations of wetting of oil (oleophilic - OL or oleophobic-OP) and water (hydrophilic-HL and hydrophobic-HP) on a surface. The four extreme wettabilities are: HP/OP (omniphobic, all liquids non-wetting), ${ }^{[1]} \mathrm{HP} / \mathrm{OL}$ (water nonwetting, oil wetting), ${ }^{[2]} \mathrm{HL} / \mathrm{OP}$ (water wetting, oil 


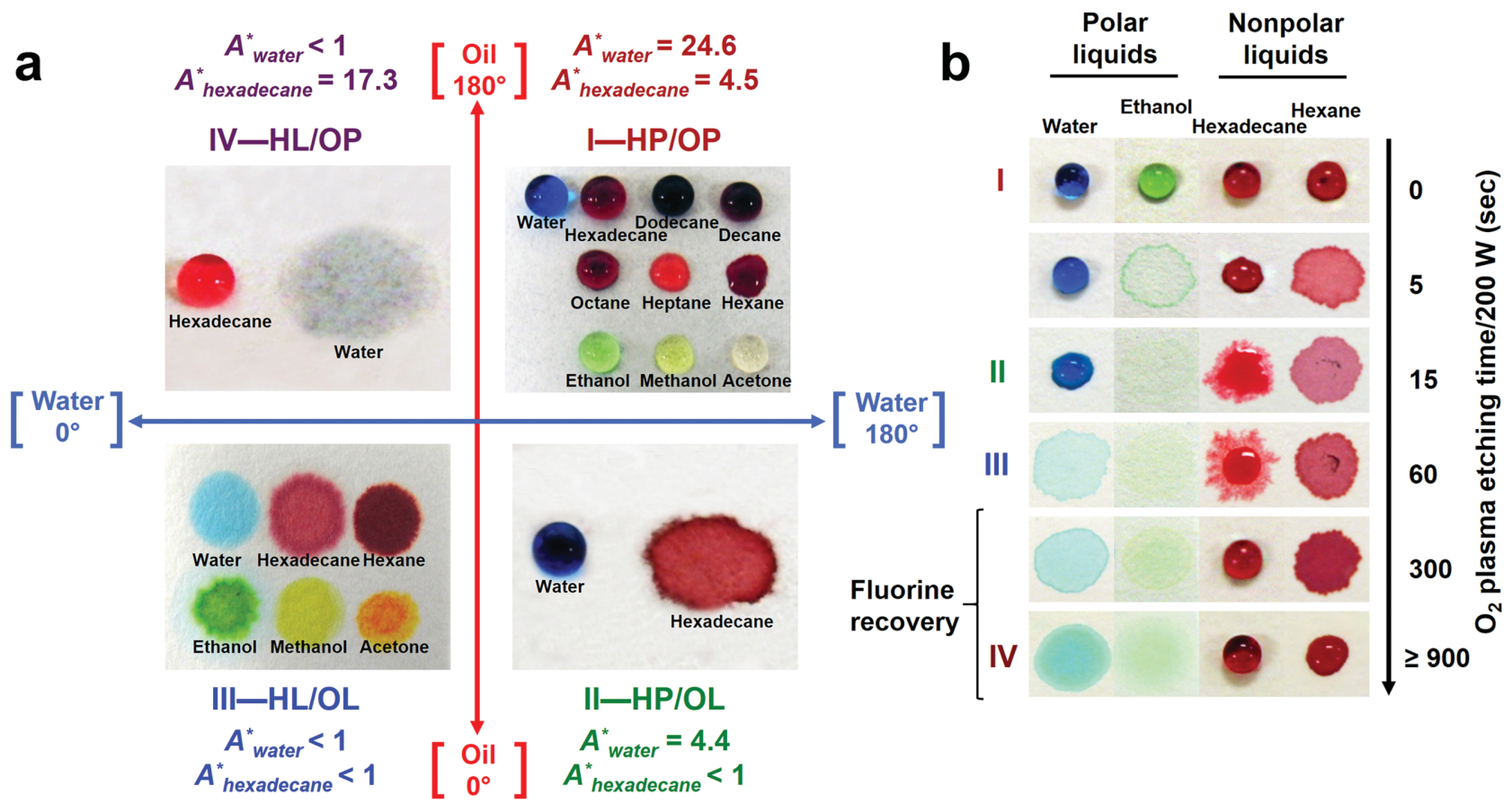

Figure 1. Paper-based surfaces with extreme wettability. a) A wettability landscape illustrating the concept of surfaces with extreme wettability. The $x$ - and $y$-axes on this plot vary from the minimum $\left(0^{\circ}\right)$ to the maximum $\left(180^{\circ}\right)$ contact angles for water and oil, respectively. Quadrant I (top right on this plot) illustrates omniphobic surfaces, i.e., surfaces that are simultaneously hydrophobic and oleophobic. Quadrant II (bottom right) illustrates hydrophobic and oleophilic surfaces. Quadrant III (bottom left) illustrates omniphilic surfaces, i.e., surfaces that are simultaneously hydrophilic and oleophilic. Quadrant IV (top left) illustrates hydrophilic and oleophobic surfaces. Values of the robustness factor $A^{*}$ on the different paper-based surfaces are also provided. b) An illustration of the changes in wettability of an omniphobic paper (fluoro-paper A) surface upon continuous exposure to $\mathrm{O}_{2}$ plasma. Short exposure time yields a hydrophobic and oleophilic surface. Further $\mathrm{O}_{2}$ plasma exposure yields an omniphilic surface. Extended etching eventually yields a hydrophilic and oleophobic surface due to fluorine recovery, as discussed in the main text. Thus, it is possible to systematically transition between surfaces with all four extreme wettabilities by simply changing the $\mathrm{O}_{2}$ plasma exposure time.

non-wetting), ${ }^{[3]}$ and HL/OL (omniphilic, all liquids wetting), ${ }^{[4]}$ as shown in Figure 1a.

In this work, we have developed a method of generating omniphobic paper surfaces that are resistant to wetting by a broad range of liquids, including numerous low surface tension solvents. Further, we have also developed a methodology to induce selective wetting of liquids with different surface tensions and polarities on a paper surface. This allows us to realize the four extreme wettabilities with oil and water, as discussed above, on paper surfaces (Figure 1a). Such selective extreme wettabilities of fluidic channels, combined with improved fluidic control, make several new applications of paper-based microfluidic devices possible. These applications rely on the separation or compartmentalization of liquids based on their surface tensions and/or polarities, and include oil-water separation, liquid-liquid extraction, droplet generation for microparticle fabrication, and the measurement of the surface tension/composition of a mixture.

\section{Background}

The engineering of liquid-repellent surfaces typically involves the manipulation of two key surface parameters: surface energy and roughness. ${ }^{[14]} \mathrm{A}$ droplet of liquid on a rough substrate can adopt one of the following two configurations to minimize its overall free energy. ${ }^{[15]}$ In the first case, the liquid droplet may completely wet a given surface, covering all of the surface asperities that are present underneath the droplet. Such complete surface coverage leads to the so-called 'fully-wetted' interface or the Wenzel state. ${ }^{[14 a]}$

On the other hand, on a sufficiently rough surface, a "composite" interface may lead to a lower overall free energy. In this case, the rough surface is not fully wetted by the liquid, and pockets of air remain trapped underneath the liquid droplet. The apparent contact angle in this state is typically calculated using the Cassie-Baxter model, ${ }^{[14 b]}$ given as

$\cos \theta^{*}=r_{\phi} \phi_{\mathrm{s}} \cos \theta-\left(1-\phi_{\mathrm{s}}\right)$

where $\theta$ is the Young's contact angle, i.e., the contact angle on a smooth non-textured surface, ${ }^{[16]} \theta^{*}$ is the apparent contact angle on a textured surface, $\phi_{\mathrm{s}}$ is the projected area of the wetted solid over the total projected area, and $r_{\phi}$ is the roughness of the wetted solid. A textured surface can yield either a fully wetted or a composite interface with a given liquid as determined by the overall free energy of the system. ${ }^{[14 d, e, 15 b, 17]} \mathrm{A}$ composite interface is required for surfaces to display very high contact angles with different liquids. We have previously shown that the presence of re-entrant texture is critical for a surface to achieve a composite interface with extremely low surface tension liquids, such as different oils and alcohols..$^{[1,2,18]}$ 
Table 1. Fabrication parameters and the resulting contact angles with water and hexadecane for each of the three different fluorinated paper substrates developed in this work.

\begin{tabular}{|c|c|c|c|c|c|c|c|c|c|}
\hline \multirow[b]{3}{*}{ Fluoro-paper } & \multirow[b]{3}{*}{ Substrate } & \multirow[b]{3}{*}{ Silanization conditions } & \multicolumn{4}{|c|}{ Contact angles $\left[{ }^{\circ}\right]$} & \multicolumn{3}{|c|}{$A^{*}$} \\
\hline & & & \multicolumn{2}{|c|}{ Water } & \multicolumn{2}{|c|}{ Hexadecane } & \multirow[b]{2}{*}{ Water } & \multirow[b]{2}{*}{ Hexadecane } & \multirow[b]{2}{*}{ Cell medium } \\
\hline & & & $\theta_{\mathrm{adv}}^{*}$ & $\theta_{\text {rec }}^{*}$ & $\theta_{\mathrm{adv}}^{*}$ & $\theta_{\text {rec }}^{*}$ & & & \\
\hline A & Filter paper & $\begin{array}{l}50^{\circ} \mathrm{C},<5 \mathrm{mmHg}, 18 \mathrm{~h} \\
\text { trichlorosilane }\end{array}$ & 161 & 143 & 136 & 103 & 24.6 & 4.5 & - \\
\hline B & Copy paper & $\begin{array}{c}70^{\circ} \mathrm{C},<5 \mathrm{mmHg}, 24 \mathrm{~h} \\
\text { trichlorosilane }\end{array}$ & 153 & 0 & 113 & 0 & 19.3 & 3.1 & - \\
\hline C & Filter paper & $\begin{array}{c}120^{\circ} \mathrm{C},<5 \mathrm{mmHg}, 24 \mathrm{~h} \\
\text { triethoxysilane }\end{array}$ & 167 & 157 & 162 & 128 & $>45.0^{\mathrm{a})}$ & 13.6 & 25.4 \\
\hline
\end{tabular}

a) No breakthrough could be observed for the minimum measurable droplet size ( $1 \mathrm{~nL})$.

The two important physical characteristics for a composite state on a textured surface with a particular contacting liquid are (i) the robustness of the composite interface to pressure perturbations and (ii) the areal fraction (1- $\phi_{s}$ in Equation (1)) of the air/vapor pockets within the composite interface, as a larger areal fraction typically yields higher contact angles with a given liquid. The breakthrough pressure $\left(P_{\mathrm{b}}\right)$ is defined as the pressure at which a given composite interface is disrupted by sagging of the liquid-vapor interface.

In our previous work, we developed a dimensionless design parameter, $A^{*}$, to predict the robustness of the composite interface, which is defined as $A^{*}=P_{\mathrm{b}} / P_{\text {ref. }} P_{\text {ref }}$ is a characteristic reference pressure, given as $P_{\text {ref }}=2 \gamma_{\mathrm{lv}} / \ell_{\text {cap }}$. Here $\ell_{\text {cap }}=\sqrt{\gamma_{\mathrm{lv}} / \rho \mathrm{g}}$, where $\gamma_{1 \mathrm{v}}$ is the surface tension of the liquid, $\rho$ is the fluid density, and $g$ is the acceleration due to gravity. As defined, $P_{\text {ref }}$ is close to the minimum pressure difference across the composite interface for millimetric or larger liquid droplets or puddles. ${ }^{[19]}$

For a texture that is dominated by periodic cylindrical features, such as the fibrous paper samples considered here, the robustness parameter $A^{*}$ may be calculated as

$A^{*}=\frac{P_{\text {breakthrough }}}{P_{\text {ref }}}=\frac{R \ell_{\text {cap }}}{D^{2}}\left(\frac{1-\cos \theta}{1+2(R / D) \sin \theta}\right)$

Here, $R$ is the fiber radius and $D$ is half of the space between adjacent cylinders. Large values of the robustness factor $\left(A^{*} \gg 1\right)$ indicate the formation of a robust composite interface, with very high breakthrough pressures, while $A^{*}<1$ predicts complete wetting of the rough interface. Our previous work has shown that the $A^{*}$ parameter does a good job of providing an a priori estimation of experimentally measured breakthrough pressure. ${ }^{[1,19]}$

\section{Results}

\subsection{Generation of All Four Extreme Wettabilities on Fluorinated Paper}

We first developed an omniphobic cellulose paper that resists wicking or spreading of most liquids, including low surface tension solvents (Figure 1a, quadrant I). This surface was prepared by fluoro-silanization of commercially available cellulose filter paper (Experimental Section). The re-entrant texture of the cellulose fibers, combined with the low surface energy of the fluoro-silane, yielded a porous omniphobic substrate, referred to as fluoro-paper A (Table 1; Figure S1, Supporting Information). The apparent advancing contact angle $\left(\theta_{\text {adv }}^{*}\right)$ of water on fluoropaper A was $>160^{\circ}$, and the robustness parameters $A^{*}{ }_{\text {water }}=24.6$ and $A_{\text {hexadecane }}^{*}=4.5$, indicated that both water and hexadecane were in a stable, non-wetted, Cassie-Baxter state (see Experimental Section for details). In addition, even very low surface tension liquids, including hexane (non-polar, $18.4 \mathrm{mN} \mathrm{m}^{-1}$, $\theta_{\mathrm{adv}}^{*}=91^{\circ}$ ), and ethanol (polar, $22.3 \mathrm{mN} \mathrm{m}^{-1}, \theta_{\mathrm{adv}}^{*}=112^{\circ}$ ) did not wet this surface (Table S2, Supporting Information).

This omniphobic fluoro-paper A was subsequently treated with $\mathrm{O}_{2}$ plasma $(200 \mathrm{~W}$, unless stated otherwise; Experimental Section) for different times to obtain surfaces that were selectively wet by different liquids depending on their surface tension and polarity. Typically, $\mathrm{O}_{2}$ plasma treatment increases the surface energy of a polymer surface through oxidation. ${ }^{[20]}$ As a consequence, the wettability of a surface generally increases with $\mathrm{O}_{2}$ plasma exposure time, with the lower surface tension liquids wetting the surface first. This trend was observed at relatively short exposure times $<60 \mathrm{~s}$ (Figure 1b; Figure S2, Supporting Information). For example, a $15 \mathrm{~s}$ application of $\mathrm{O}_{2}$ plasma transformed the originally omniphobic fluoro-paper $\mathrm{A}$ into a $\mathrm{HP} / \mathrm{OL}$ surface $\left(A_{\text {water }}^{*}=4.4, A_{\text {hexadecane }}^{*}<1\right)$. Low surface tension liquids like alkanes and ethanol wetted the surface, while high surface tension liquids like water remained non-wetting. A further increase in $\mathrm{O}_{2}$ plasma etching time to $60 \mathrm{~s}$ yielded an omniphilic surface, on which even a high surface tension liquid like water completely wets $\left(A_{\text {water }}^{*}\right.$ and $\left.A_{\text {hexadecane }}^{*}<1\right)$ (Figure 1b).

This initial enhancement in surface wettability is mainly due to the simultaneous defluorination and oxidation of the fluoropaper A surface. This is evident from X-ray photoelectron spectroscopy (XPS) analysis of the surface chemistry (Figure S3e,f, Supporting Information). For example, after a short burst (5 s) of $\mathrm{O}_{2}$ plasma, a dramatic increase in the surface oxygen content can be observed. Increasing the $\mathrm{O}_{2}$ plasma etching time to $30 \mathrm{~s}$ yields the minimum value for the fluorine to oxygen $(\mathrm{F} / \mathrm{O})$ ratio. In addition to analyzing the changes in surface chemistry, scanning electron microscopy (SEM) was used to observe the development of fibrillar nanostructures along the cellulose microfibers (Figure S3a-c, Supporting Information). 
This nano-texturing occurs due to the selective etching of amorphous components within the cellulose fibers. ${ }^{[21]}$ After $60 \mathrm{~s}$ of etching, the increased solid surface energy, and increased surface roughness, transform the originally omniphobic fluoropaper A surface into an omniphilic material.

Counter-intuitively, exposure to $\mathrm{O}_{2}$ plasma for an even longer time yields the HL/OP extreme wettability (Figure 1b; Figure S2, Supporting Information). When the $\mathrm{O}_{2}$ plasma etching time was $>300 \mathrm{~s}$, the surface became non-wettable by hexadecane, and when it was $>900 \mathrm{~s}$, the surface became non-wettable even by hexane $\left(A^{*}{ }_{\text {water }}<1, A_{\text {hexadecane }}^{*}=17.3\right)$. Note that the $A^{*}$ value for hexadecane on fluoro-paper A after $900 \mathrm{~s}$ of etching is even greater than that on the original omniphobic surface. It was found that after the F/O ratio reaches its minimum value at around $30 \mathrm{~s}$ of etching, the ratio begins to increase again (Figure S3e,f, Supporting Information). This fluorine recovery may be attributed to the redeposition of fluorocarbon fragments present in the vacuum chamber after the fluorosilane is removed during the initial stages. ${ }^{[22]}$ Simultaneously, the extended etching also causes the surface of the cellulose microfibers to be significantly roughened due to the development of fibrillar nanostructures (Figure S3d, Supporting Information). Through a synergistic effect from both enhanced roughness and recovered fluorine content, the surfaces eventually resist wetting by low surface tension alkanes (e.g., $\theta_{\text {adv }}^{*}$ for hexadecane is $154^{\circ}$ on fluoro-paper A etched for $900 \mathrm{~s}$ ).

Surfaces that repel low surface tension liquids almost always repel water as well, due to its higher surface tension. However, the partially fluorinated, extremely rough surface produced by extended etching yields a reconfigurable or "hygro-responsive" surface, similar to those we have reported previously. ${ }^{[3]}$ When a polar liquid like water contacts this HL/OP surface, the surface reconfigures to enable the polar hydroxyl groups along the cellulose fibers to hydrogen bond with the water molecules. This interaction/surface reconfiguration does not occur with non-polar liquids, and the result is a surface that can be wet by water, but not by non-polar liquids like hexane. Overall, by simply changing the time a fluoro-paper A surface is exposed to $\mathrm{O}_{2}$ plasma, we can create surfaces with all four extreme wettabilities (Figure 1b). Further, in our testing, the different surfaces maintained their extreme wettabilities for several days following fabrication.

\subsection{Generation of 2D and 3D Fluidic Channels on Fluoro-Paper A}

2D microfluidic channels with defined dimensions and wettabilities were initially fabricated by selectively covering fluoropaper A substrates with polyimide tape prior to $\mathrm{O}_{2}$ plasma etching (Figure S4, Supporting Information; Experimental Section). Seven different polar and nonpolar liquids (surface tensions from $\gamma_{\mathrm{v}}=18.4-72.8 \mathrm{mN} \mathrm{m}^{-1}$ ) were placed into channels exposed for 5-900 s (Figure S5a, Supporting Information), and their maximum wetting length (Table S3, Supporting Information), average wetting velocity (total wetting length over total time ${ }^{[23]}$ ) (Table S4, Supporting Information), and wetting depth (Table S5, Supporting Information) were measured (Supporting Information). Both the maximum wetting length and the peak average wetting velocity for each liquid occurred at different plasma etching times. This is consistent with the selective variation in wettability with etching time as shown in Figure $1 \mathrm{~b}$. Additionally, only the upper surface of the etched paper sample became more wettable, as directional $\mathrm{O}_{2}$ etching did not penetrate deep into the paper. This enables the confinement of liquid to a limited depth, reduces liquid retention within the channels, limits unproductive sample consumption, and also permits the fabrication of multi-layer flow devices on a single paper substrate, as discussed below.

Next, we fabricated 3D flow channels on a single piece of fluoro-paper A that were capable of confining liquids with a wide range of surface tensions and polarities (Experimental Section; Supporting Information). As shown in Figure 2a, eight fluidic channels were arranged orthogonally on a device,
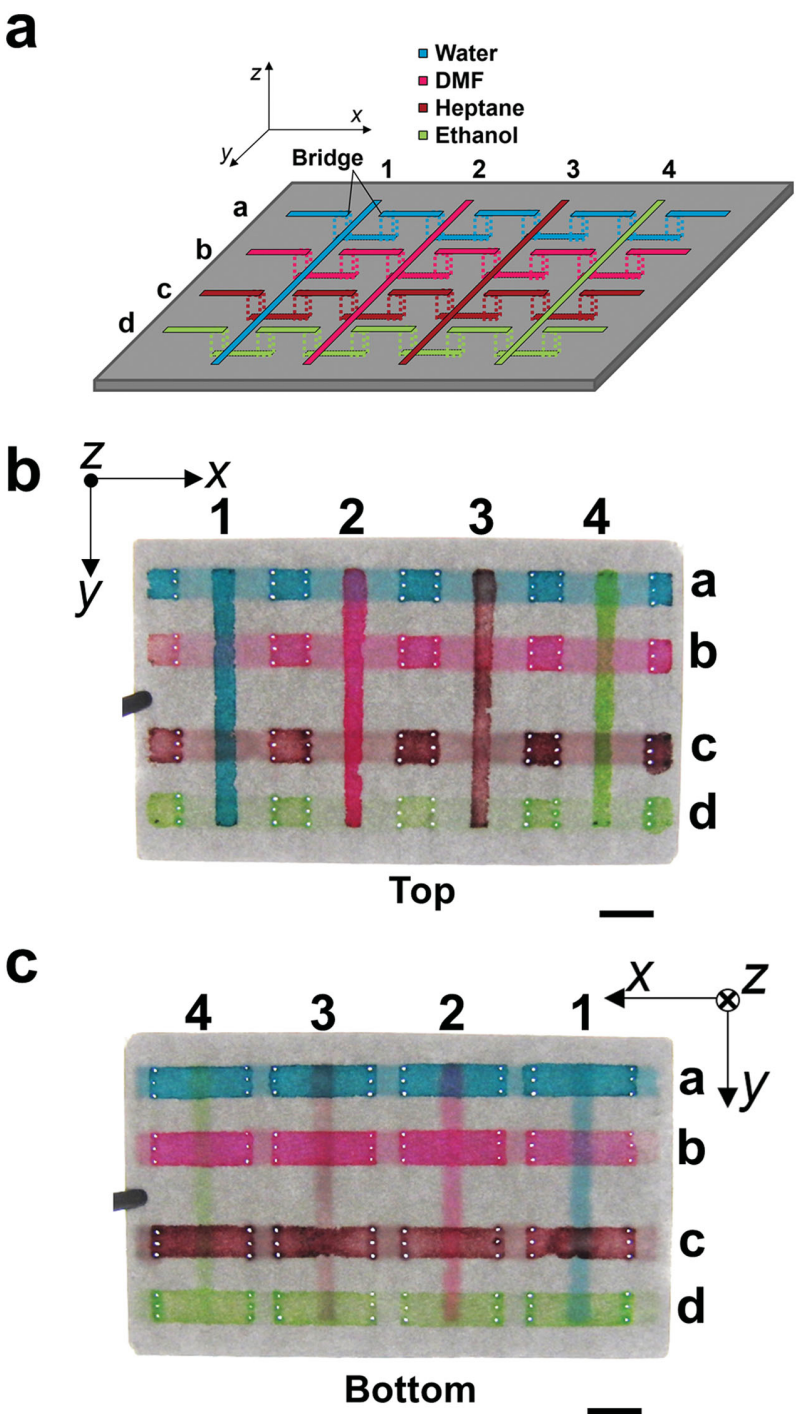

Figure 2. 3D paper device. a) A schematic illustrating the 3D patterning of fluidic channels on a single piece of paper, by incorporating channels on both sides of a fluoro-paper A substrate. Two groups of channels are patterned orthogonally in the $x$ - and $y$-directions. These are labeled as $a$, b, c, $d$ and 1, 2, 3, 4, respectively. b,c) The top and bottom of a 3D paper device after being filled with four test liquids: water (blue), DMF (red), heptane (brown) and ethanol (green). Scale bars are $5 \mathrm{~mm}$. 
with four channels each along the $x$ - and $y$-directions. To traverse from one edge of the device to the other, liquids filling the channels along the $x$-direction must bridge straight channels in the $y$-direction by flowing to the bottom surface of the paper. This is accomplished by passing the liquid through short bridge channels on the bottom surface using arrays of vertical perforations (500 $\mu \mathrm{m}$ diameter) penetrating the entire thickness of the paper at the junctions, then returning it to the top surface via more perforations (Figure S6, Supporting Information).

The channels were selectively $\mathrm{O}_{2}$ plasma etched for different times at $350 \mathrm{~W}$ to obtain maximum flow rates for each of the four liquids used (Experimental Section). Approximately $20 \mu \mathrm{L}$ droplets of water, dimethylformamide (DMF), ethanol, and heptane were loaded at the ends of the channels. Complete filling of the $4 \times 4$ bridge device was observed for water and DMF, but ethanol and heptane had to be filled from both ends to counteract their higher evaporation rates (Movie S1, Supporting Information). No mixing between test liquids in the separate $x$ or $y$-direction channels was observed at any point (Figure $2 \mathrm{~b}, \mathrm{c}$ ). Overall, these 3D channels can increase the available interfacial area for the patterning of channels, facilitate routing of channels in complex devices, and enable the incorporation of different assays on different sides of paper-based microfluidic devices. ${ }^{[10]}$

\subsection{Printing of Fluidic Channels on Fluoro-Paper B}

To increase the ease of use, and reduce the time required for the patterning process, we also developed a mask-free, direct printing technique to produce fluidic channels of arbitrary geometry, capable of containing very low surface tension liquids (Figure 3a; Experimental Section). To enable such direct printing of fluidic channels, we first developed a second type of fluorinated-paper, fluoro-paper B, which exhibits high advancing contact angles, but zero receding contact angles with both water and oil (Table 1; Figure S1, Supporting Information).
While the hysteresis on this surface was significantly higher than on the fluoro-paper A surface, the $A^{*}$ values remain $\gg 1$, indicating that the paper still strongly resists wetting by all liquids $\left(A_{\text {water }}^{*}=19.3, A_{\text {hexadecane }}^{*}=3.1\right)$. The zero receding contact angles, and very high advancing contact angles, enabled the facile deposition of defect-free films of a high surface energy polyurethane adhesive by preventing the dewetting and spreading of the adhesive. The films were deposited by directly applying a viscous solution of the polyurethane adhesive through a tapered deposition nozzle attached to an XYZ motorized stage (Experimental Section). The minimum width of the printed lines, and the interstitial spacing between lines, were $700 \mu \mathrm{m}$ and $400 \mu \mathrm{m}$, respectively. A line drawing of the Lurie Bell Tower at the University of Michigan $(5.3 \times 4.4 \mathrm{~cm})$ was successfully printed on fluoro-paper B and filled with a low viscosity (5 cSt) silicone oil $\left(\gamma_{\mathrm{v}}=19.8 \mathrm{mN} \mathrm{m}^{-1}\right)$ (Figure 3b-e, Movie S2, Supporting Information).

\subsection{Continuous Oil-Water Separation on Fluoro-Paper A}

Next, we focused on multiple novel applications of paper-based microfluidic devices that necessitate the utilization of surfaces with extreme wettabilities, which would not have been possible with simple omniphilic channels patterned on an omniphobic background. These applications, including liquid-liquid extraction, and droplet generation by flow focusing, require the selective confinement of oil and water phases and the maintenance of a stable organic-aqueous liquid-liquid interface. To verify this selectivity in wettability, and the stability of the oil-water interface, we first fabricated an oil-water separation device consisting of a HP/OL channel placed head-to-head against a HL/ OP channel on fluoro-paper A (Figure 4a; Figure S7, Supporting Information). Heptane was pre-mixed with water by pumping at $100 \mu \mathrm{L} \mathrm{min}{ }^{-1}$ through a stainless steel mesh, and the mixture was continuously added onto the central reservoir containing the interface between the HP/OL and HL/OP channels. The
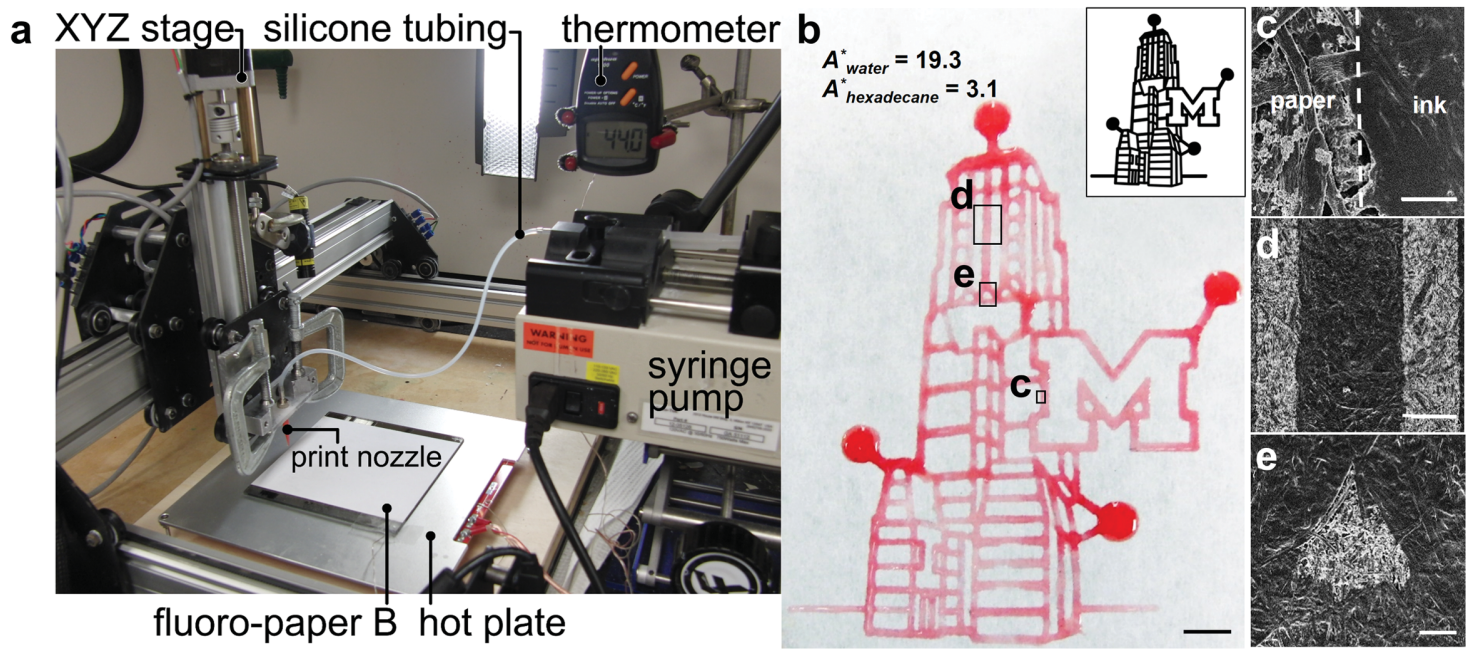

Figure 3. Mask-free, direct printing of fluidic channels on omniphobic paper. a) The printing apparatus. b) A printed pattern on a fluoro-paper B surface. The channels have been filled with silicone oil $\left(\gamma_{\mathrm{v}}=19.8 \mathrm{mN} \mathrm{m}{ }^{-1}\right)$, dyed red. The inset is the $5.3 \times 4.4 \mathrm{~cm}$ pattern being printed. Scale bar is $5 \mathrm{~mm}$. c-e) SEM images highlighting the magnified texture for different areas on the patterned surface shown in (b) after the channel is filled with silicone oil. The corresponding areas are labeled as c, d, and e in (b). Scale bars are $50 \mu \mathrm{m}$ for c), $500 \mu \mathrm{m}$ for d), and $200 \mu \mathrm{m}$ for e). 

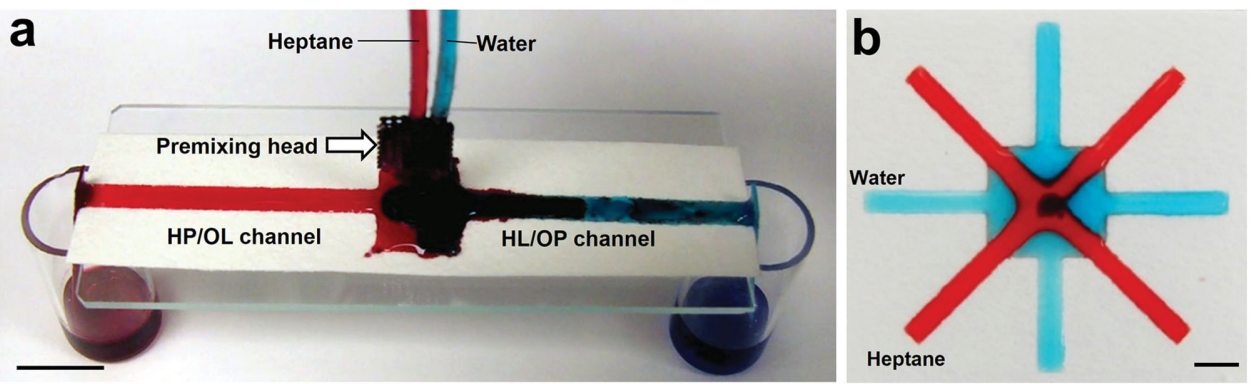

Figure 4. Surface oil-water separation and dispensing. a) Continuous oil-water separation device. A HP/OL channel is combined with an HL/OP channel on a fluoro-paper A surface and used to separate a mixture (50\% by volume) of heptane and water. Scale bar is $1 \mathrm{~cm}$. b) Multiplexed oil-water dispenser, composed of four HL/OP and four HP/OL channels radially arranged from a central reservoir. Any oil or water droplets when added to this reservoir are automatically split and dispensed into four HP/OL or HL/OP channels, respectively. Scale bar is $5 \mathrm{~mm}$.

heptane-water mixture spontaneously separated as the heptane selectively wet the HP/OL channel, while the water selectively wet the HL/OP channel. Pure heptane (>99.9\%) and water $(>99.9 \%)$ were collected at the opposite ends of the device. With this approach, continuous separation of an oil-water mixture could be realized on a paper substrate, based solely on the difference in wettabilities of the patterned channels. To demonstrate the scalability of this approach, we also fabricated a multiplexed oil-water dispenser with four outlets each for both oil and water (Figure 4b; Experimental Section). Continuous separation and dispensing of a heptane-water mixture could be readily achieved with this device (Movie S3, Supporting Information).

\subsection{Continuous Liquid-Liquid Extraction on Fluoro-Paper A}

We also demonstrated continuous liquid-liquid extraction on a paper-based microfluidic device. Liquid-liquid extraction is a well-known unit operation in chemical engineering, used to extract one or more miscible components from a liquid mixture by exposing it to another liquid with preferential solubility for the target components. It is also widely utilized in analytical chemistry, biology, and the environmental sciences. It is particularly well suited for lab-on-a-chip based purification and analytical units. ${ }^{[24]}$

We fabricated a liquid-liquid extraction device consisting of a HP/OL channel that is placed alongside a HL/OP channel on fluoro-paper A (Figure 5a; Figure S8a, Supporting Information). The feed was a mixture of octane and ethanol (dyed with methylene blue, which is preferentially soluble in ethanol), and the extractant was water. The flow rate of the feed and the extractant were both $50 \mu \mathrm{L} \mathrm{min}{ }^{-1}$ to maintain a 1:1 volume ratio. The HL/OP channel was first filled with water. The water was completely confined in the channel and did not overflow into the background or the adjacent HP/OL channel. The octaneethanol mixture was subsequently introduced into the $\mathrm{HP} / \mathrm{OL}$ channel, yielding a well-defined and stable organic-aqueous liquid-liquid interface (Figure 5b). Ethanol was continuously extracted by the water stream, moving from the top channel to the bottom channel. The extractant (water) started out transparent in the inlet tube and at the beginning of the extraction zone, but became blue, due to the presence of the blue-dyed ethanol, by the time it reached the end of the HL/OP channel.
In comparison, the feed (octane + ethanol) started out blue in the extraction zone and became clear by the time it reached the end of the HP/OL channel, due to the near-complete removal of dyed ethanol. After the system reached steady state, the three zones (extraction, transition, and clear) in the HP/OL channel were clearly observed.

In this experiment, the majority of the ethanol $(\approx 82 \%)$ was removed from the feed. This was evaluated by performing refractive index measurements on the feed and the extractant

a



b

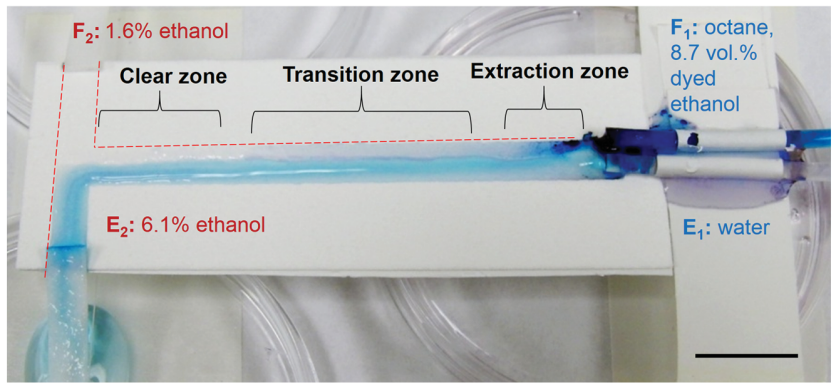

Figure 5. Surface liquid-liquid extraction. a) A schematic highlighting the design of a device that enables continuous, co-current, liquid-liquid extraction on paper. b) A HP/OL channel is combined with an HL/OP channel, side-by-side, on a fluoro-paper A surface and used for liquidliquid extraction. The bottom channel is HL/OP. This channel is filled with water, which serves as the extractant. The top channel is $\mathrm{HP} / \mathrm{OL}$ and is filled with a mixture of octane and ethanol. Scale bar is $1 \mathrm{~cm}$. 
before flowing through the device, as well as on the raffinate (feed minus extracted material) and the extract (extractant mixture with extracted material) produced downstream. The compositions were determined by comparing these refractive index values with calibration curves generated using mixtures of a known composition (Figure S8b,c, Supporting Information). Note that in this case, the movement of ethanol from the organic (octane) phase to the aqueous phase occurred due to the imbalance in chemical potential of ethanol across the liquid-liquid interface. A control experiment was performed with the same geometry but with adjacent omniphilic channels (Figure S9, Supporting Information). Without the selectivity in wettability between the organic and aqueous phases, the organic phase, due to its lower surface tension, will always undercut and displace the aqueous phase, causing the formation of an irregular organic-aqueous liquid-liquid interface. This prevented successful liquid-liquid extraction.

\subsection{Surface Emulsification for Particle Fabrication on Fluoro-Paper A}

Paper-based microfluidic devices reported in the literature are mainly designed and used for analysis and/or detection, rather than fabrication or synthesis. ${ }^{[10 f]}$ We now discuss the development of an open-channel paper-based microfluidic device to fabricate polymeric microparticles. The fabricated device performs droplet emulsification using a "flow-focusing" geometry, previously only possible with traditional closed-channel devices. ${ }^{[25]}$

Emulsification in a microfluidic device is performed to produce discrete droplets of a target substance with highly controlled, uniform dimensions at high production rates. Among the numerous applications of this technique are the production of polymeric micro- and nanoparticles, parallel experimentation with reactions occurring in droplets, which may be individually controlled, and encapsulation of single cells for analysis. ${ }^{[26]}$ Droplet microfluidics can also lower the overall volume required for effective sample transport through a device and prevent waste due to wetting of the microfluidic substrate, as a scarce or expensive target fluid may be suspended in an inexpensive, abundant carrier fluid. ${ }^{[27]}$ Microfluidic emulsification performed in an open channel may also be preferable for a number of reasons. It is simpler to add samples and re-agents to specific locations on an open-channel device, the devices are less prone to clogging, and they have a large liquid-vapor interfacial area. Open channels also have a reduced hydraulic resistance, so less applied pressure is required for flow. This in turn leads to less applied shear stress on potentially sensitive samples such as encapsulated cells. ${ }^{[28]}$

The functionality of our open-channel emulsification device again relies on the selective wetting of adjacent HP/OL and HL/ OP channels, and their ability to stabilize an organic-aqueous, liquid-liquid interface (Figure 6a; Figure S10, Supporting Information; Experimental Section). An aqueous surfactant solution was introduced within the HL/OP outer channel. The HP/OL inner channel was then filled with a UV cross-linkable precursor, immiscible with water. Both the aqueous and organic phases remained within their respective channels as they were
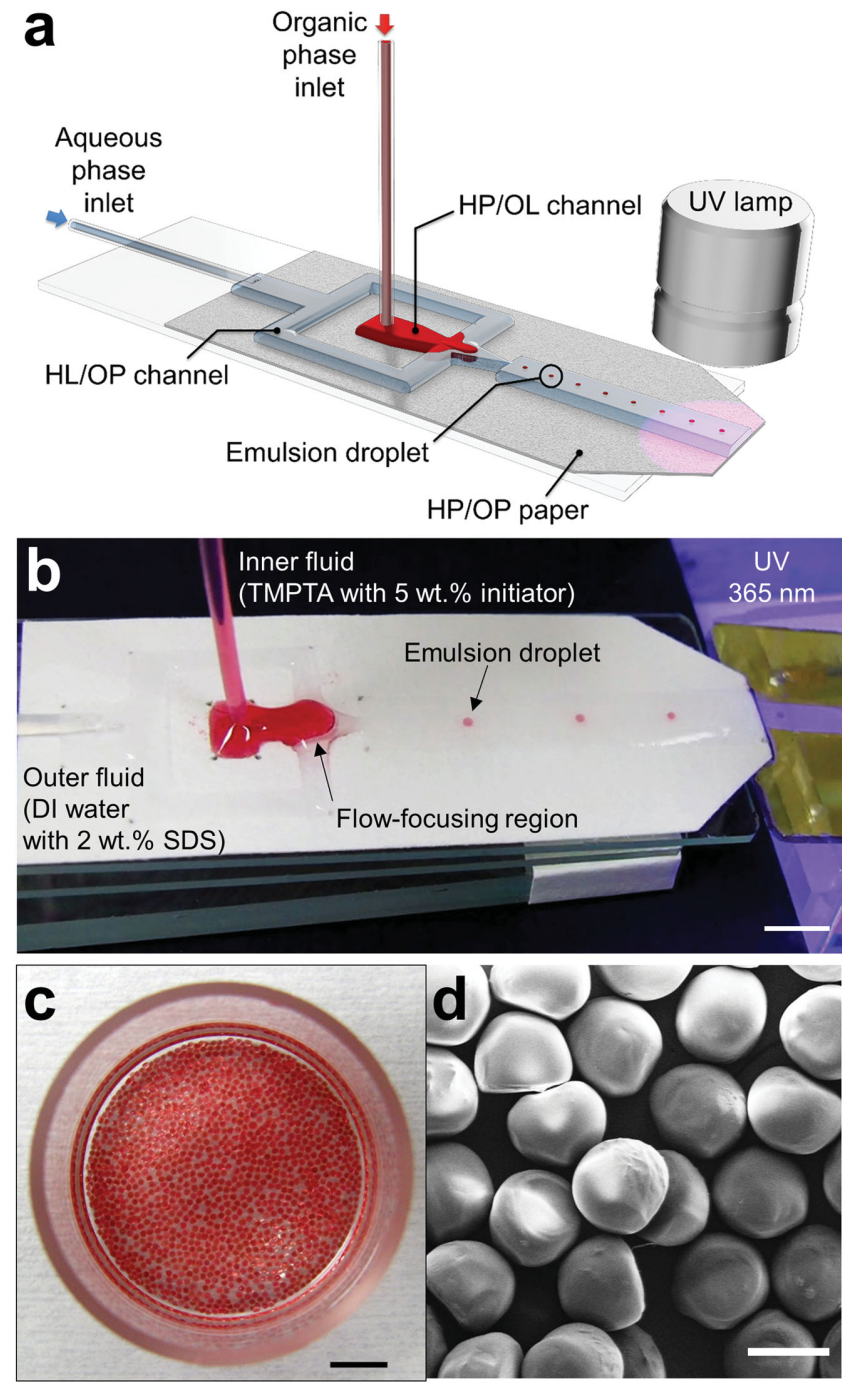

Figure 6. Paper-based device for microparticle fabrication. a) A schematic highlighting the design of a flow-focusing geometry for microfluidic emulsification and micro-particle fabrication on paper. b) The micro-particle fabrication device on a fluoro-paper A surface illustrated in (a). Scale bar is $5 \mathrm{~mm}$. c) A photograph of the cross-linked micro-particles collected after $1 \mathrm{~h}$ of operation (particles are dyed red and collected in a glass vial). Scale bar is $5 \mathrm{~mm}$. d) An SEM image of the UV cross-linked microparticles. Scale bar is $500 \mu \mathrm{m}$.

pumped through the device, which was crucial in achieving stable emulsification (droplets of the organic phase suspended within the continuous aqueous phase) using the flow-focusing geometry (Figure 6b).

Over a thousand micro-particles could be collected within $1 \mathrm{~h}$ from a single-surface emulsification device (Figure 6c, Movie S4, Supporting Information). After crosslinking by passing under a UV lamp, the prepared polymeric microparticles were collected and found to be hemispheres $(\approx 500 \mu \mathrm{m}$ base diameter) (Figure 6d). This geometry is attributed to the fact that the precursor droplets (organic phase) float on top of the aqueous phase in the open channel. Multiple parallel devices could easily be fabricated on the paper surface to increase the production rate. 


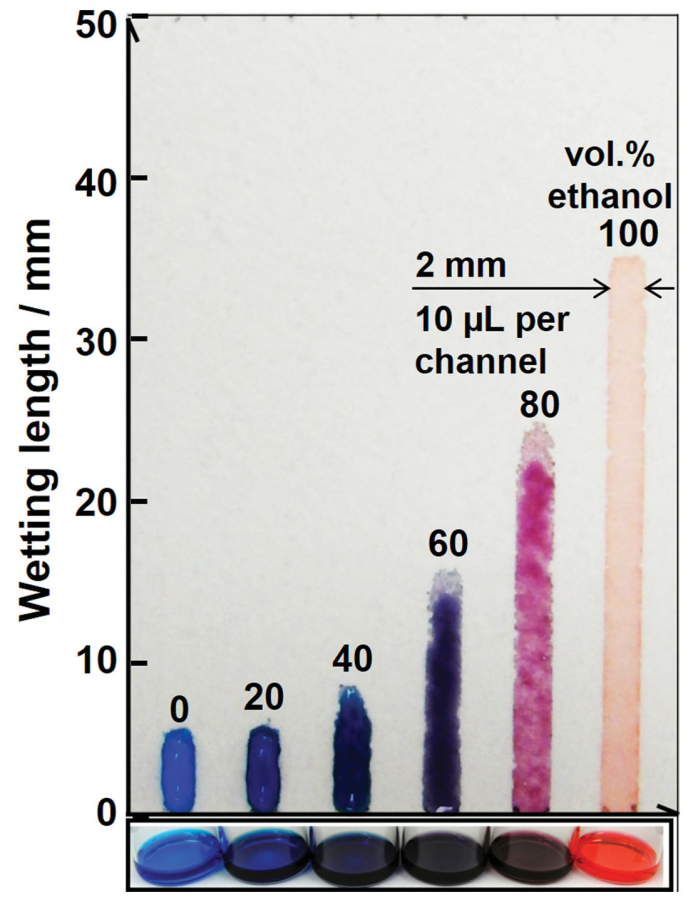

Figure 7. Measurement of the surface tension/composition of mixtures. A paper-based alcoholmeter developed on a fluoro-paper A surface. The alcoholmeter consists of patterned $\mathrm{HP} / \mathrm{OL}$ channels on an omniphobic background.

\subsection{Surface Tension/Composition Measurement Using Fluoro-Paper A}

The developed microfluidic devices can also be useful for the facile determination of a binary mixture's composition. As an example, we have developed a so-called "alcohol-meter," that can readily determine the volume composition of an unknown ethanol-water mixture. Ethanol-water mixtures of varying composition were placed within a set of parallel HP/OL channels on fluoro-paper A (Figure 7). The wetting length in these channels increases with decreasing liquid surface tension, i.e., the wetting length increased with increasing ethanol concentration. ${ }^{[29]}$ By measuring the maximum liquid wetting length for any unknown ethanol-water mixture, it is easy to determine its surface tension, and therefore its composition, by comparing to wetting lengths of mixtures with known compositions. The developed device could similarly be calibrated for use with a range of different binary mixtures.

\subsection{Ovarian Cancer Cell Microarrays on Fluoro-Paper C}

Finally, we investigated biological applications of the developed paper-based microfluidic devices. In particular, we developed a paper-based device that could be used for generating patterned arrays of mammalian cells. Patterned cell arrays can be used for the inexpensive, high-throughput, testing of different bioactive substances such as drugs, oligonucleotides, and
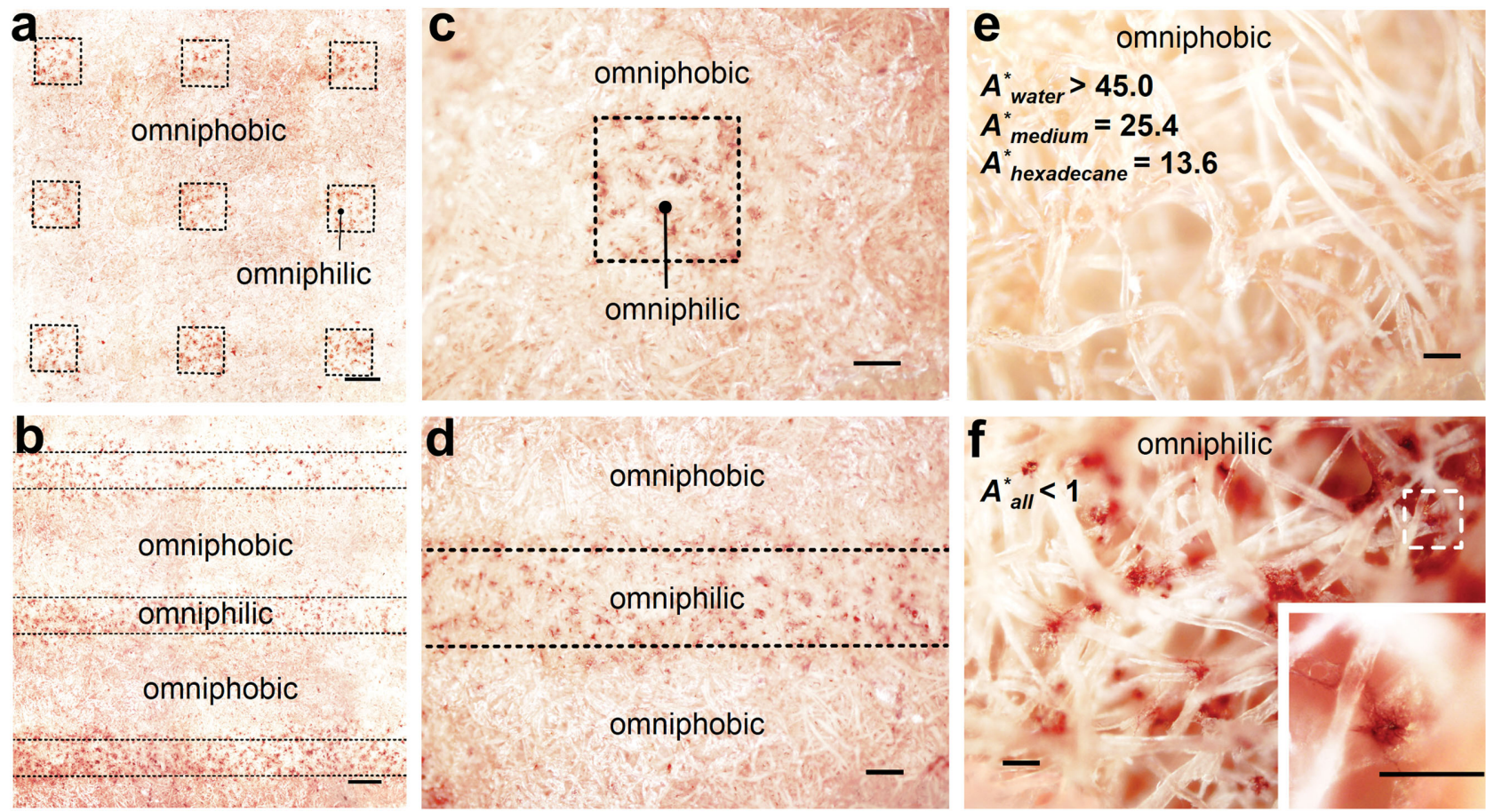

Figure 8. Generation of cell microarrays on surfaces with patterned wettability. a-f) Growth of OVCAR3 (ovarian cancer) cells on an omniphobic (fluoro-paper C) surface patterned with omniphilic domains. This paper has a high $A^{*}$ value for the growth culture medium used, ensuring longevity of the non-wetted state while immersed. The cells preferentially grow on the omniphilic regions and not on the omniphobic background. Scale bars are a,b) $500 \mu \mathrm{m}, \mathrm{c}, \mathrm{d}) 200 \mu \mathrm{m}$, and e,f) $50 \mu \mathrm{m}$. 
peptides. ${ }^{[28 c]}$ For this application, in order to limit cell adhesion to the background, we developed a third type of fluorinated paper, fluoro-paper C. It exhibits higher contact angles and lower contact angle hysteresis with both water and hexadecane than fluoro-paper A, as well as, higher $A^{*}$ values $\left(A_{\text {water }}^{*}>45.0\right.$, $\left.A_{\text {hexadecane }}^{*}=13.6\right)$ (Table 1 ; Figure S1, Supporting Information). This indicates that it has a significantly higher resistance to wetting. This was required to prevent the wetting of the omniphobic channels during the extended immersion under cell growth medium, required as part of the cell culture process. The fluoro-paper $\mathrm{C}$ fabrication involves the creation of a hierarchical texture of cellulose nanofibrils on filter paper with extended $\mathrm{O}_{2}$ plasma etching prior to fluorosilanization (Experimental Section). The fluoro-paper $\mathrm{C}$ was then patterned with omniphilic square spots and channels $(\approx 500 \mu \mathrm{m}$ wide) by exposure to $\mathrm{O}_{2}$ plasma through porous stainless steel masks (Figure S11, Supporting Information). These patterned omniphobic substrates with omniphilic domains were subsequently exposed to OVCAR3 cells (human ovarian adenocarcinoma cell line) in supplemented growth medium for $10 \mathrm{~h}$, and then stained with iodonitrotetrazolium chloride (Experimental Section). The OVCAR3 cells selectively adhered to and grew on the omniphilic domains as opposed to the omniphobic background (Figure 8).

\section{Conclusion}

In summary, we have developed paper-based microfluidic devices that demonstrate, for the first time, all four possible combinations of extreme wettabilities with water and different oils. The fabricated devices allow for the selective localization, manipulation, and transport of virtually all high- and low-surface tension liquids based on differences in surface tension and/or polarity. The developed designs are compatible with both 2D lateral flow, and 3D multilayer flow, and can also be fabricated using a mask-free, direct printing technique. Finally, by enabling the generation of stable, immiscible organic-aqueous liquid-liquid interfaces in open channels, the fabricated devices greatly expand the potential applications of paper-based microfluidic devices in a wide range of fields such as oil-water separation, chemical analysis, purification, chemical/particle synthesis, and high-throughput testing of different bioactive substances.

\section{Experimental Section}

Preparation of Fluorinated Paper Substrates: Substrates were either cellulose filter papers (GE Life Sciences, Whatman Grade 3, pore size $6 \mu \mathrm{m}$, thickness $390 \mu \mathrm{m}$ ) or cellulose copy paper (Boise X-9 Multiuse OX9001, OfficeMax). Fluorosilanes, (heptadecafluoro1,1,2,2-tetrahydrodecyl trichloro-silane and heptadecafluoro-1,1,2,2tetrahydrodecyl triethoxy-silane), were purchased from Gelest. Asahiklin AK-225 (dichloropentafluoropropane) was purchased from SPI Supplies. All of the materials and reagents were used as received. See Table 1 for a summary of the three types of fluoro-paper developed in this manuscript.

For fluoro-paper A, each batch of cellulose filter papers was treated with trichlorosilane $\left(400 \mu \mathrm{L}\right.$ per $400 \mathrm{~cm}^{2}$, an excess quantity) by vapor phase deposition in a vacuum oven (Precision Scientific, $50{ }^{\circ} \mathrm{C}$, $<5 \mathrm{mmHg}$ ) for $18 \mathrm{~h}$ (Figure S1, Supporting Information, fluoro-paper A). After fluorosilanization, the paper was soaked in AK-225 for $24 \mathrm{~h}$ to remove the unreacted fluorosilanes. Fluoro-paper A is omniphobic (Table S2, Supporting Information).

For fluoro-paper B, the substrate was copy paper, and the treatment method was similar to that of fluoro-paper A (Figure S1, Supporting Information, fluoro-paper $\mathrm{B}$ ), but at a higher temperature, and longer time $\left(70{ }^{\circ} \mathrm{C}, 24 \mathrm{~h}\right)$. Compared to fluoro-paper A, fluoro-paper B has flattened fibers resulting in reduced overall roughness, which yielded receding contact angles close to zero. However, advancing contact angles with both water and hexadecane remained high (Table 1).

For fluoro-paper $\mathrm{C}$, the substrates were filter papers, pre-treated by $\mathrm{O}_{2}$ plasma etching with a Plasmatherm 790 (chamber vacuum 20 mTorr, $\mathrm{O}_{2}$ flow rate $20 \mathrm{sccm}$, bias RF source power $200 \mathrm{~W}$, etching time $10 \mathrm{~min}$ ) to produce nanoscale fibrillar texture. The silanization process was similar to that for fluoro-paper A, but at a higher temperature, and for a longer duration $\left(120^{\circ} \mathrm{C}, 24 \mathrm{~h}\right)$ (Figure S1, Supporting Information fluoro-paper (C). The triethoxy functional silane was used instead of the trichloro functional silane to avoid embrittlement of the paper (already weakened by extended plasma etching) by the hydrochloric acid byproduct produced during extended high temperature reactions with chloro-silanes. The micro- and nanoscale hierarchical texture due to the pre-functionalization etching made fluoro-paper $C$ omniphobic, with significantly lower contact angle hysteresis than fluoro-paper A (Table 1).

Test Liquids and Dyes: Deionized water was generated with a Pall Corporation Cascada RO-water purification system. Other test liquids: DMF, chloroform, acetone, methanol, ethanol, and alkanes ( $n$-hexane, $\mathrm{n}$-heptane, n-octane, $\mathrm{n}$-decane, $\mathrm{n}$-dodecane, $\mathrm{n}$-hexadecane), were all purchased from Fisher Scientific (Table S1, Supporting Information). Solvent Blue 38, Disperse Red 1, Methylene Blue Indicator (MBI) (Sigma Aldrich), and food coloring (McCormick) were used to dye polar liquids. Oil Red O (Alfa Aesar) was used to dye non-polar liquids.

Masking of Fluorinated Paper Surface and Selective $\mathrm{O}_{2}$ Plasma Treatment to Produce Microfluidic Devices: Fluoro-paper A was masked by Kapton polyimide adhesive tape $(3.175,6.35$, or $12.5 \mathrm{~mm}$ wide, $25.4 \mu \mathrm{m}$ thick, purchased from ULINE) by manual application. The masked paper was selectively $\mathrm{O}_{2}$ plasma etched (Plasmatherm 790, chamber vacuum $20 \mathrm{mTorr}, \mathrm{O}_{2}$ flow rate $20 \mathrm{sccm}$, bias RF source power $200 \mathrm{~W}-350 \mathrm{~W}$ ) to obtain functional devices (Figure S4, Supporting Information). Plasma etching time was varied to obtain the desired wettability in each channel. The maximum wetting length, average wetting velocity, and wetting depth were characterized for a range of liquids for different times in simple 2D channels (Note 1; Table S3-5, Supporting Information). Functional devices for oil-water separation, liquid-liquid extraction, droplet emulsification, and surface tension measurement were produced by this masked $\mathrm{O}_{2}$ plasma etching method and are described in the main text.

To fabricate a 3D paper device, both sides were masked in the desired geometry, and arrays of $500 \mu \mathrm{m}$ diameter perforations were manually introduced at the appropriate locations to allow liquid to transfer to the opposite side of the paper. $\mathrm{O}_{2}$ plasma etching was applied onto both sides to make the channels wettable to the target liquids, with varying times to obtain maximum wettability for each target liquid. The $\mathrm{O}_{2}$ plasma etching was optimized for channels with one, two, and four bridges for each target liquid, with increasing time and power required for more bridges (Note 2; Figure S6, Supporting Information; Figure 2). For four bridges, the optimal etching times were: $180 \mathrm{~s}$ for water channels, $60 \mathrm{~s}$ for DMF channels, $30 \mathrm{~s}$ for heptane channels, and $15 \mathrm{~s}$ for ethanol channels, all with the plasma power set at $350 \mathrm{~W}$. Movie S1 (Supporting Information) shows the channels with four bridges being filled.

Printed Channels on Fluoro-Paper B: A mask-free patterning method to generate channels was developed by directly depositing high surface energy material on fluoro-paper B. Compared to fluoro-paper A, the nearly zero receding contact angles for both water and oil on the fluoropaper B guaranteed good contact and adhesion between the ink and the paper surface, with no dewetting during application. Meanwhile, 
the retention of high advancing contact angles prevented the ink from further wicking or spreading on the surface of fluoro-paper B.

The ink was a water-based polyurethane (Aleene's Fabric Fusion permanent fabric adhesive), dissolved in ethanol to make a $500 \mathrm{mg} \mathrm{mL}^{-1}$ solution, and then diluted $1: 3$ by volume with a $20 \mathrm{mg} \mathrm{mL}^{-1}$ sodium dodecyl sulfate aqueous solution. The final solution was probe sonicated (Heat Systems Ultrasonic Processor Sonicator) for $2 \mathrm{~min}$ and filtered through a $0.2 \mu \mathrm{m}$ syringe filter. This material formed uniform, colorless films, with minimal dewetting (related to surface tension, viscosity, and evaporation rate of the ink), excellent adherence to the paper, and was wet by most liquids other than water.

The ink was selectively applied to the paper with a lab-made printing apparatus (Figure $3 \mathrm{a}$ ). The printing head is a tapered polypropylene dispensing nozzle (24 gauge or $570 \mu \mathrm{m}$ outer diameter, McMaster-Carr) connected via silicone tubing (1/16" inner diameter, McMaster-Carr) to a syringe pump (KD Scientific Model 200) operating at $1 \mathrm{~mL} \mathrm{~h} \mathrm{~h}^{-1}$. A motorized XYZ motion stage (Shapeoko 2) allows selective deposition. The motion of each axis was computer-controlled, as was the syringe pump. The fluoro-paper B substrates were temporarily attached to glass plates with a spray adhesive, which were then mounted to a carefully leveled hot plate held at $45^{\circ} \mathrm{C}$

Patterns for the XYZ platform were generated by drawing them in vector graphics software (Inkscape) and then converting them to $\mathrm{G}$-code commands. The pattern chosen was a line drawing of the Lurie Bell Tower at the University of Michigan, with an overall size of $5.3 \mathrm{~cm} \times 4.4 \mathrm{~cm}$. When $\approx 25 \mu \mathrm{L}$ of low viscosity silicone oil (dyed with Oil Red O) was placed in each of the four reservoirs, the whole pattern was completely filled, with wetting driven only by capillary forces (Movie S2, Supporting Information)

Surface Emulsification on Fluoro-Paper A: An outer HL/OP channel (200 $\mathrm{W} \mathrm{O}_{2}$ plasma etched, $300 \mathrm{~s}$ ) was integrated with an inner $\mathrm{HP} /$ $\mathrm{OL}$ channel $\left(200 \mathrm{~W} \mathrm{O}_{2}\right.$ plasma etched, $\left.15 \mathrm{~s}\right)$. The common boundary between the channels was designed to be a flow-focusing nozzle (Figure S10, Supporting Information). The HL/OP channel was filled with a continuously flowing carrier fluid (2 wt\% aqueous solution of sodium dodecyl sulfate, purchased from Hoefer). The $\mathrm{HP} / \mathrm{OL}$ channel was filled with a continuously flowing crosslinkable polymer (1,1-trimethylolpropane triacrylate (TMPTA, purchased from Polysciences) with $5 \mathrm{wt} \%$ initiator (2-hydroxy-2-methylpropiophenone, purchased from Sigma Aldrich)). The geometry of the two channels and the flow rates of the precursor and carrier fluids $\left(1500\right.$ and $5 \mu \mathrm{L} \mathrm{min}{ }^{-1}$, respectively, controlled by independent syringe pumps (KD Scientific KDS-230)) forced the formation of monodisperse droplets of the UV crosslinkable precursor in the carrier fluid (Movie S4, Supporting Information). These droplets were carried downstream to a UV lamp (UVP BLAK-RAY B-100SP, $140 \mathrm{~W}, 365 \mathrm{~nm}$ ), which caused them to crosslink and solidify. The particles were then collected and imaged.

Patterning of OVCAR3 Cancer Cells on Fluoro-Paper C: All tissue culture reagents were purchased from Life Technologies (Carlsbad, CA) unless specified otherwise. The growth medium used was phenol red free RPMI 1640 supplemented with $10 \%$ fetal bovine serum, 1× L-Glutamine and $1 \times$ antibiotics/antimycotics. Human ovarian adenocarcinoma cell line, $\mathrm{NIH}$ :OVCAR3, was purchased from ATCC (Manassas, VA).

Fluoro-paper $C$ substrates were covered with porous stainless steel masks with patterns of squares $(500 \mu \mathrm{m} \times 500 \mu \mathrm{m}, 1.5 \mathrm{~mm}$ spacing $)$ and stripes (500 $\mu \mathrm{m}$ wide, $1.5 \mathrm{~mm}$ spacing) purchased from Metal Etch Services. Omniphilic domains were obtained on an omniphobic background with $\mathrm{O}_{2}$ plasma etching (Plasmatherm 790, chamber vacuum $20 \mathrm{mTorr}, \mathrm{O}_{2}$ flow rate $20 \mathrm{sccm}$, bias RF source power $30 \mathrm{~W}$ for $2 \mathrm{~min}$ ) (Figure S11, Supporting Information). The patterned paper was sterilized by exposure to a $254 \mathrm{~nm}$ germicidal UV lamp for $2 \mathrm{~h}$.

Cells were cultured in growth medium until $\approx 80 \%$ confluency and then trypsinized per regular passage. Counts were performed with a hemocytometer and 0.75 million cells were plated on each paper sample with $5 \mathrm{~mL}$ growth medium in a six-well plate. The samples were incubated for $10 \mathrm{~h}$ at $37{ }^{\circ} \mathrm{C}$ with $5 \% \mathrm{CO}_{2}$ and then stained with iodonitrotetrazolium chloride (Sigma-Aldrich). Samples were rinsed once with $1 \times$ Dulbecco's phosphate-buffered saline (DPBS) and allowed to air dry prior to imaging with an Olympus BX-51 visible light microscope.

Characterization Methods: Photographs and videos were recorded with a digital camera (Casio EX-F1). SEM images were acquired with a Hitachi SU8000 ultra-high resolution SEM with an accelerating voltage of $10 \mathrm{kV}$. XPS analysis was conducted using a Kratos Axis Ultra XPS. The monochromatic Al-K $\alpha$ X-ray source was operated at $15 \mathrm{kV}$ and $10 \mathrm{~mA}$. Photoelectrons were collected at a takeoff angle of $\approx 65^{\circ}$ relative to the sample surface. Wide-scan survey spectra were acquired at an analyzer pass energy of $160 \mathrm{eV}$ and a step size of $1 \mathrm{eV}$. O 1s, F 1s and C 1s highresolution spectra were collected at an analyzer pass energy of $60 \mathrm{eV}$ and a step size of $1 \mathrm{eV}$.

All contact angle measurements were obtained using a RaméHart 200-Fl goniometer. $3 \mu \mathrm{L}$ droplets were used for static contact angle measurement. The advancing and receding contact angles measurements were performed starting with a $3 \mu \mathrm{L}$ droplet on the surface and then growing and shrinking it by adding and removing a small volume $(\approx 2 \mu \mathrm{L})$ using a $2 \mathrm{~mL}$ micrometer syringe (Gilmont) while continually measuring contact angles. Contact angle hysteresis was calculated as the difference between advancing and receding contact angles. At least three measurements were performed on each sample surface. Typical error in measurements was $\pm 2^{\circ}$.

To measure $A^{*}$, a series of droplets of varying volume were put on sample surfaces. A higher Laplace pressure can be expected from a smaller droplet due to the increase in curvature. ${ }^{[30]} A$ decrease in the apparent contact angle of droplet will result from an increase in Laplace pressure as it causes the composite interface to sag deeper into the texture. The apparent contact angle was measured with the goniometer. If the apparent contact angle was below $90^{\circ}$ with a specific droplet volume, the droplet was considered to have wetted the surface, and the corresponding Laplace pressure considered to be greater than the breakthrough pressure for the surface. $A^{*}$ can then be calculated as the Laplace pressure divided by the reference pressure (see main text).

\section{Supporting Information}

Supporting Information is available from the Wiley Online Library or from the author.

\section{Acknowledgements}

The authors thank the National Science Foundation and the Nanomanufacturing program for supporting this work through grant \#1351412. The authors thank Dr. Ki-Han Kim and the Office of Naval Research (ONR) for financial support under grant N00014-12-1-0874. The authors also thank Dr. Charles Y. Lee and the Air Force Office of Scientific Research (AFOSR) for financial support under grant FA955015-1-0329. This work was performed in part at the University of Michigan Lurie Nanofabrication Facility.

Received: April 12, 2016

Revised: May 29, 2016

Published online: June 22, 2016

[1] A. Tuteja, W. Choi, J. M. Mabry, G. H. McKinley, R. E. Cohen, Proc. Natl. Acad. Sci. USA 2008, 105, 18200.

[2] A. Tuteja, W. Choi, M. Ma, J. M. Mabry, S. A. Mazzella, G. C. Rutledge, G. H. McKinley, R. E. Cohen, Science 2007, 318, 1618.

[3] A. K. Kota, G. Kwon, W. Choi, J. M. Mabry, A. Tuteja, Nat. Commun. 2012, 3, 1025.

[4] S. P. Kobaku, A. K. Kota, D. H. Lee, J. M. Mabry, A. Tuteja, Angew. Chem. 2012, 124, 10256; Angew. Chem. Int. Ed. 2012, 51, 10109. 
[5] a) A. K. Kota, W. Choi, A. Tuteja, MRS Bull. 2013, 38, 383; b) G. Kwon, E. Post, A. Tuteja, MRS Commun. 2015, 5, 475.

[6] J. P. Rolland, D. A. Mourey, MRS Bull. 2013, 38, 299.

[7] A. W. Martinez, S. T. Phillips, M. J. Butte, G. M. Whitesides, Angew. Chem. 2007, 119, 1340; Angew. Chem. Int. Ed. 2007, 46, 1318.

[8] a) P. N. Nge, C. I. Rogers, A. T. Woolley, Chem. Rev. 2013, 113, 2550; b) A. W. Martinez, S. T. Phillips, G. M. Whitesides, E. Carrilho, Anal. Chem. 2010, 82, 3; c) A. W. Martinez, Bioanalysis 2011, 3 , 2589; d) X. Li, D. R. Ballerini, W. Shen, Biomicrofluidics 2012, 6, 11301; e) D. M. Cate, J. A. Adkins, J. Mettakoonpitak, C. S. Henry, Anal. Chem. 2015, 87, 19.

[9] a) E. Carrilho, A. W. Martinez, G. M. Whitesides, Anal. Chem. 2009, 81, 7091; b) Z. Nie, C. A. Nijhuis, J. Gong, X. Chen, A. Kumachev, A. W. Martinez, M. Narovlyansky, G. M. Whitesides, Lab Chip 2010, 10, 477; c) X. Li, J. Tian, T. Nguyen, W. Shen, Anal. Chem. 2008, 80, 9131; d) X. Li, J. Tian, G. Garnier, W. Shen, Colloids Surf., B 2010, 76, 564; e) J. Olkkonen, K. Lehtinen, T. Erho, Anal. Chem. 2010, 82, 10246; f) D. M. Cate, W. Dungchai, J. C. Cunningham, J. Volckens, C. S. Henry, Lab Chip 2013, 13, 2397; g) M. Santhiago, J. B. Wydallis, L. T. Kubota, C. S. Henry, Anal. Chem. 2013, 85, 5233.

[10] a) A. W. Martinez, S. T. Phillips, G. M. Whitesides, Proc. Natl. Acad. Sci. USA 2008, 105, 19606; b) A. W. Martinez, S. T. Phillips, Z. Nie, C. M. Cheng, E. Carrilho, B. J. Wiley, G. M. Whitesides, Lab Chip 2010, 10, 2499; c) G. G. Lewis, M. J. DiTucci, S. T. Phillips, Angew. Chem. 2012, 124, 12879; Angew. Chem. Int. Ed. 2012, 51, 12707; d) K. M. Schilling, D. Jauregui, A. W. Martinez, Lab Chip 2013, 13, 628; e) H. Liu, R. M. Crooks, J. Am. Chem. Soc. 2011, 133, 17564; f) L. Ge, S. Wang, X. Song, S. Ge, J. Yu, Lab Chip 2012, 12, 3150 .

[11] a) A. K. Yetisen, M. S. Akram, C. R. Lowe, Lab Chip 2013, 13, 2210 ; b) J. L. Osborn, B. Lutz, E. Fu, P. Kauffman, D. Y. Stevens, P. Yager, Lab Chip 2010, 10, 2659; c) E. Fu, S. A. Ramsey, P. Kauffman, B. Lutz, P. Yager, Microfluid. Nanofluid. 2011, 10, 29; d) P. Spicar-Mihalic, B. Toley, J. Houghtaling, T. Liang, P. Yager, E. Fu, J. Micromech. Microeng. 2013, 23, 067003.

[12] P. Lisowski, P. K. Zarzycki, Chromatographia 2013, 76, 1201.

[13] a) A. C. Glavan, R. V. Martinez, A. B. Subramaniam, H. J. Yoon R. M. D. Nunes, H. Lange, M. M. Thuo, G. M. Whitesides, Adv. Funct. Mater. 2013, 24, 60; b) J. Wang, M. R. Monton, X. Zhang, C. D. Filipe, R. Pelton, J. D. Brennan, Lab Chip 2014, 14, 691; c) W. J. Lan, E. J. Maxwell, C. Parolo, D. K. Bwambok, A. B. Subramaniam, G. M. Whitesides, Lab Chip 2013, 13, 4103.

[14] a) R. N. Wenzel, Ind. Eng. Chem. 1936, 28, 988; b) A. B. D. Cassie, S. Baxter, Trans. Faraday Soc. 1944, 40, 0546; c) M. Callies, D. Quéré, Soft Matter 2005, 1, 55; d) M. Nosonovsky, Langmuir 2007, 23, 3157; e) A. Marmur, Langmuir 2003, 19, 8343; f) D. Quéré, Physica A 2002, 313, 32.

[15] a) R. Shuttleworth, G. L. J. Bailey, Discuss. Faraday Soc. 1948, 3, 16; b) R. E. Johnson, R. H. Dettre, in Contact Angle, Wettability, and Adhesion, Vol. 43 (Ed: F. M. Fowkes), American Chemical Society, Washington, D.C. 1964, 112

[16] T. Young, Philos. Trans. R. Soc. London 1805, 95, 65.

[17] N. A. Patankar, Langmuir 2003, 19, 1249.

[18] S. Pan, A. K. Kota, J. M. Mabry, A. Tuteja, J. Am. Chem. Soc. 2013, 135, 578.

[19] W. Choi, A. Tuteja, S. Chhatre, J. M. Mabry, R. E. Cohen G. H. McKinley, Adv. Mater. 2009, 21, 2190.

[20] J. N. Lai, B. Sunderland, J. M. Xue, S. Yan, W. J. Zhao, M. Folkard, B. D. Michael, Y. G. Wang, Appl. Surf. Sci. 2006, 252, 3375.

[21] Y. L. Lam, C. W. Kan, C. W. M. Yuen, J. Appl. Polym. Sci. 2012, 124, 3341.

[22] M. Morra, E. Occhiello, F. Garbassi, Surf. Interface Anal. 1990, 16, 412

[23] T. M. Schutzius, M. Elsharkawy, M. K. Tiwari, C. M. Megaridis, Lab Chip 2012, 12, 5237.

[24] P. Mary, V. Studer, P. Tabeling, Anal. Chem. 2008, 80, 2680.

[25] a) A. S. Utada, L. Y. Chu, A. Fernandez-Nieves, D. R. Link, C. Holtze, D. A. Weitz, MRS Bull. 2007, 32, 702; b) S. L. Anna, N. Bontoux, H. A. Stone, Appl. Phys. Lett. 2003, 82, 364.

[26] a) S. Y. Teh, R. Lin, L. H. Hung, A. P. Lee, Lab Chip 2008, 8, 198; b) H. N. Joensson, H. A. Svahn, Angew. Chem. 2012, 124, 12342; Angew. Chem. Int. Ed. 2012, 51, 12176.

[27] M. G. Simon, A. P. Lee, in Microdroplet Technology: Principles and Emerging Applications in Biology and Chemistry, (Eds: P. Day, A. Manz, Y. Zhang), Springer-Verlag, New York 2012, p. 23.

[28] a) B. Zhao, J. S. Moore, D. J. Beebe, Science 2001, 291, 1023; b) G. V. Kaigala, R. D. Lovchik, E. Delamarche, Angew. Chem. 2012, 124, 11386; Angew. Chem. Int. Ed. 2012, 51, 11224; c) E. Ueda, P. A. Levkin, Adv. Mater. 2013, 25, 1234

[29] G. Vasquez, E. Alvarez, J. M. Navaza, J. Chem. Eng. Data 1995, 40, 611.

[30] H.-J. Butt, K. Graf, M. Kappl, Physics and Chemistry of Interfaces, Wiley-VCH, Weinheim, Germany 2013. 